\title{
AVALIAÇÃO DE QUATRO ALTERNATIVAS DE ANÁLISE DE EXPERIMENTOS EM LÁTICE QUADRADO, QUANTO À ESTIMAÇÃO DE COMPONENTES DE VARIÂNCIA ${ }^{(1)}$
}

\author{
HEYDER DINIZ SILVA ${ }^{(2)}$; DANIEL FURTADO FERREIRA ${ }^{(3)}$; \\ CLESO ANTÔNIO PATTO PACHECO ${ }^{(4)}$
}

\begin{abstract}
RESUMO
Estudou-se, no presente trabalho, a eficiência das seguintes alternativas de análise de experimentos realizados em látice quanto à precisão na estimação de componentes de variância, através da simulação computacional de dados: i) análise intrablocos do látice com tratamentos ajustados (primeira análise); ii) análise do látice em blocos casualizados completos (segunda análise); iii) análise intrablocos do látice com tratamentos não-ajustados (terceira análise); iv) análise do látice como blocos casualizados completos, utilizando as médias ajustadas dos tratamentos, obtidas a partir da análise com recuperação da informação interblocos, tendo como quadrado médio do resíduo a variância efetiva média dessa análise do látice (quarta análise). Os resultados obtidos mostram que se deve utilizar o modelo de análise intrablocos de experimentos em látice para se estimarem componentes de variância sempre que a eficiência relativa do delineamento em látice, em relação ao delineamento em Blocos Completos Casualizados, for superior a $100 \%$ e, em caso contrário, deve-se optar pelo modelo de análise em Blocos Casualizados Completos. A quarta alternativa de análise não deve ser recomendada em qualquer das duas situações.

Palavras chave: látice, componentes de variância, eficiência, simulação.
\end{abstract}

\section{ABSTRACT \\ EVALUATION OF FOUR ALTERNATIVES OF ANALYSIS OF EXPERIMENTS IN SQUARE LATTICE, WITH EMPHASIS ON ESTIMATE OF VARIANCE COMPONENT}

The efficiency of fur alternatives of analysis of experiments in square lattice, related to the estimation of variance components, was studied through computational simulation of data: i) intrablock analysis of the lattice with adjusted treatments (first analysis); ii) lattices analysis as a randomized complete blocks design (second analysis); iii); intrablock analysis of the lattice with non-adjusted treatments (third analysis); iv) lattice analysis as a randomized complete blocks design, using the adjusted means of treatments, obtained through the analysis of lattice with recuperation of interblocks information, having as the residual mean square, the average effective variance of this same lattice analysis (fourth analysis). The results showed that the intrablock model, must be used with adjusted treatments in lattice experiments to estimate variance components every time that the relative efficiency of the lattice design, relatively to the randomized complete blocks design be upper to $100 \%$, and in the opposite case the randomized complete blocks design model must be used. The fourth alternative of analysis must not be recommended in both situations.

Key words: lattice designs, variance componets, effciency, simulation.

(1) Recebido para publicação em 20 de julho de 1999 e aceito em 16 de março de 2000.

(2) Departamento de Matemática, Universidade Federal de Uberlândia (UFU), Campus Santa Mônica, 38400-902 Uberlândia (MG). E-mail: heyder@ufu.br

(3) Departamento de Ciências Exatas, Universidade Federal de Lavras, Caixa Postal 37, 37200-000 Lavras (MG).

(4) CNPMS-EMBRAPA, Caixa Postal 151, 35701-970 Sete Lagoas (MG). 


\section{INTRODUÇÃO}

Nos programas de melhoramento vegetal é comum a avaliação de um grande número de tratamentos (linhagens, progênies, híbridos etc.). Com o objetivo de controlar a heterogeneidade ambiental, os estatísticos normalmente recomendam o delineamento em blocos casualizados completos. No entanto, nessa situação de grande número de tratamentos ou nas situações onde se utilizam parcelas de grande tamanho, esse delineamento perde a sua eficiência, uma vez que a pressuposição de homogeneidade dentro dos blocos é geralmente violada. Em tal situação, o melhorista deve optar por um tipo de delineamento que possua maior controle local, como os delineamentos em blocos incompletos, e, dentre estes, os látices quadrados (square lattice) têm-se destacado, sendo muito utilizados na experimentação agronômica, principalmente no melhoramento genético vegetal.

Segundo Pimentel-Gomes (1990), o delineamento em látice foi proposto por YATES (1936) para a análise estatística de experimentos com muitos tratamentos, nos quais a eficiência do controle local pode tornar-se reduzida se forem estabelecidos blocos completos muito grandes.

Se um experimento é conduzido em látice, existem algumas alternativas de análise que podem ser realizadas. Quando o efeito de tratamentos for fixo, tendo como interesse testar hipóteses a respeito de combinações lineares dos mesmos, o problema de se escolher entre uma ou outra alternativa de análise torna-se simples. Quando, porém, o efeito de tratamentos for aleatório, naturalmente o interesse incidirá na estimação de componentes da variâncias e covariâncias, que são de grande importância no melhoramento genético vegetal, visto que o método de melhoramento e a população a serem utilizados dependem do conhecimento de parâmetros genéticos. Nesse caso, para estimação dos componentes de variância existem determinadas alternativas, porém em algumas delas o problema é mais complexo, principalmente no que concerne à análise conjunta de experimentos.

Assim, procurou-se, neste trabalho, avaliar as seguintes alternativas de análise de experimentos conduzidos em látice, quanto à precisão na estimação de componentes de variância, através da simulação computacional de dados: i) análise intrablocos do látice com tratamentos ajustados (primeira análise); ii) análise do látice como blocos casualizados completos (segunda análise); iii) análise intrablocos do látice com tratamentos não-ajustados (terceira análise); iv) análise do látice como blocos casualizados completos, utilizando as médias ajustadas dos tratamentos, obti- das a partir da análise com recuperação da informação interblocos, tendo como quadrado médio do resíduo a variância efetiva média desta mesma análise do látice (quarta análise).

\section{MATERIAL E MÉTODOS}

Para avaliação das alternativas de análise de experimentos conduzidos em látice, apresentadas neste trabalho, quanto à precisão na estimação de componentes da variância, simularam-se 4.000 experimentos no delineamento em látice $10 \times 10$, sendo $2.000 \mathrm{em}$ látice simples (duas repetições) e os outros $2.000 \mathrm{em}$ látice triplo (três repetições). Consideraram-se duas situações para a realização das simulações: a primeira levou em conta a alta eficiência relativa do látice em relação ao delineamento em blocos casualizados completos, e a segunda, a baixa eficiência relativa do látice, ou seja, com componente de variância paramétrico de blocos igual a zero.

Na simulação dos experimentos utilizou-se o seguinte modelo estatístico:

$$
\mathrm{y}_{\mathrm{i}(\mathrm{j})}=\mu+\mathrm{t}_{\mathrm{i}}+\mathrm{r}_{\mathrm{j}}+\left(\frac{\mathrm{b}}{\mathrm{r}}\right)_{\ell(\mathrm{j})}+\mathrm{e}_{\mathrm{i}(\mathrm{j})}
$$

no qual

$\begin{array}{ll}\text { yi } \ell \text { j }) \quad & \text { é o valor observado do tratamento i } \\ & (\mathrm{i}=1,2, \ldots, \mathrm{v}=100), \text { no bloco incompleto } \ell \\ & (\ell=1,2, \ldots, \mathrm{k}=10), \text { da repetição } \mathrm{j} \\ & (\mathrm{j}=1,2, \ldots, \mathrm{r}=2, \text { ou } 3) ; \\ \mu & \begin{array}{l}\text { é uma constante inerente a todas as } \\ \text { observações; }\end{array} \\ \mathrm{t}_{\mathrm{i}} & \text { é o efeito do tratamento } \mathrm{i} ; \\ \mathrm{r}_{\mathrm{j}} & \text { é o efeito da repetição j; } \\ (\mathrm{b} / \mathrm{r}) \ell(\mathrm{j}) \quad & \text { é o efeito do bloco incompleto } \ell \text { dentro da } \\ & \text { repetição j; } \\ \mathrm{e}_{\mathrm{i} \ell(\mathrm{j})} & \text { É o erro aleatório associado à observação } \\ & \text { yi }(\mathrm{j})\end{array}$

Os efeitos de tratamentos foram simulados com distribuição normal de média $\phi$ e variância $\hat{\sigma}_{\mathrm{g}}^{2}$ e obtidos por $t_{i}=\phi^{-1}\left(U_{i}\right) . \sigma_{g}, i=1,2, \ldots, 100$ em que $\phi^{-1}$ representa a distribuição normal inversa (DACHs, 1988) e $\mathrm{U}_{\mathrm{i}}$, um número aleatório com distribuição uniforme $(0,1)$. Procedimento semelhante foi utilizado na simulação dos demais efeitos, ou seja: $r_{j}=\phi^{-1}\left(U_{j}\right) . \sigma_{r}, j=1,2$ ou 3 para os látices simples e triplo, respectivamente; $(\mathrm{b} / \mathrm{r})_{\left.\ell_{\mathrm{j}}\right)}=\phi^{-1}\left(\mathrm{U}_{\left.\ell_{\mathrm{j}}\right)}\right) \cdot \sigma_{\ell_{(\mathrm{j})},} \ell=1,2$,

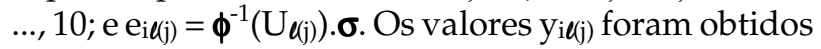
somando os efeitos simulados de acordo com o modelo anteriormente apresentado. Os parâmetros utilizados na simulação dos dados foram: 
i) $\mu=165 \mathrm{~cm} ; \sigma_{\mathrm{g}}^{2}=195 \mathrm{~cm}^{2} ; \sigma_{\mathrm{r}}^{2}=17 \mathrm{~cm}^{2} ; \sigma^{2}{ }_{\ell(j)}=$ $110 \mathrm{~cm}^{2}$ e $\sigma^{2}=95 \mathrm{~cm}^{2}$, para os experimentos com alta eficiência do látice; e

ii) $\mu=165 \mathrm{~cm} ; \sigma_{\mathrm{g}}^{2}=195 \mathrm{~cm}^{2} ; \sigma_{\mathrm{r}}^{2}=17 \mathrm{~cm}^{2} ; \sigma^{2}{ }_{(j)}=$ $0 \mathrm{~cm}^{2}$ e $\sigma^{2}=95 \mathrm{~cm}^{2}$, para os experimentos com baixa eficiência do látice.

Após simulados os experimentos, obtiveram-se as estimativas dos componentes de variância, devido a efeitos de tratamentos $\left(\hat{\sigma}_{\mathrm{g}}^{2}\right)$, residual $\left(\hat{\sigma}^{2}\right)$ e de blocos dentro de repetições $\left(\hat{\sigma}_{b}^{2}\right)$, considerando-se os seguintes modelos para análise de experimentos conduzidos em látice:

\subsection{Análise intrablocos com tratamentos ajustados e blocos dentro de repetições não-ajustados - primeira análise}

$$
y_{i}(j)=\mu+t_{i}+r_{j}+\left(\frac{b}{r}\right)_{\ell j}+e_{i \ell(j)}
$$

no qual

\begin{tabular}{|c|c|}
\hline$y i(j)$ & $\begin{array}{l}\text { é o valor observado do tratamento } \mathrm{i} \\
(\mathrm{i}=1,2, \ldots, \mathrm{v}=100) \text {, no bloco incompleto } \ell \\
(\ell=1,2, \ldots, \mathrm{k}=10) \text {, da repetição } \mathrm{j} \\
(\mathrm{j}=1,2, \ldots, \mathrm{r}=2, \text { ou } 3)\end{array}$ \\
\hline$\mu$ & $\begin{array}{l}\text { é uma constante inerente a todas as } \\
\text { observações; }\end{array}$ \\
\hline $\mathrm{T}_{\mathrm{i}}$ & é o efeito do tratamento i; \\
\hline $\mathrm{R}_{\mathrm{j}}$ & é o efeito da repetição j; \\
\hline$\left.(\mathrm{b} / \mathrm{r}) \ell_{\mathrm{j}}\right)$ & $\begin{array}{l}\text { é o efeito do bloco incompleto } \ell \text { dentro da } \\
\text { repetição j; }\end{array}$ \\
\hline$\left.e_{i} \ell j\right)$ & $\begin{array}{l}\text { é o erro aleatório associado à observação } \\
\text { yi (q) }\end{array}$ \\
\hline
\end{tabular}

É a seguinte a decomposição ortogonal da soma de quadrados de parâmetros, devida ao ajuste do modelo completo, denotada por $\mathrm{R}(\mu, \tau, \alpha, \beta)$, apresentada por VIANA (1993):

$$
\begin{array}{r}
R(\mu, \tau, \alpha, \beta)=R(\mu)+R(\alpha / \mu)+R(\beta / \mu, \alpha)+ \\
R(\tau / \mu, \alpha, \beta)
\end{array}
$$

Para a obtenção das esperanças matemáticas dos quadrados médios, apresentadas no quadro 1, foram adotadas as seguintes pressuposições:
a) $t_{i} \sim \operatorname{NID}\left(0, \sigma^{2}\right)$;
b) $\mathrm{r}_{\mathrm{j}} \sim \operatorname{NID}\left(0, \sigma_{\mathrm{r}}^{2}\right)$;
c) $(\mathrm{b} / \mathrm{r})_{\left.\ell_{\mathrm{j} j}\right)} \sim \operatorname{NID}\left(0, \sigma^{2} \mathrm{~b}\right)$;
d) $\mathrm{e}_{\mathrm{i}(\mathrm{j})} \sim \operatorname{NID}\left(0, \sigma^{2}\right)$; e
e) $t_{i}, r_{j},(b / r)_{\ell(j)}$ e e $\left.e_{i} \ell_{j}\right)$ são independentes.

A partir do quadro 1, foram obtidos os estimadores dos componentes de variância, dados por:

$$
\begin{aligned}
& \hat{\sigma}^{2}=Q_{1} \\
& \hat{\sigma}_{\mathrm{g}}^{2}=\frac{Q_{2}-Q_{1}}{\mathrm{r}\left(\frac{\mathrm{k}}{\mathrm{k}+1}\right)}
\end{aligned}
$$

e

$$
\hat{\sigma}_{\mathrm{b}}^{2}=\frac{\mathrm{Q}_{3}-\left(\frac{\mathrm{k}+1}{\mathrm{rk}}\right)\left(\mathrm{Q}_{2}-\mathrm{Q}_{1}\right)-\mathrm{Q}_{1}}{\mathrm{k}}
$$

\subsection{Análise do látice como blocos casualizados completos} - segunda análise

$$
y_{i j}=\mu+t_{i}+r_{j}+e_{i j}
$$

em que

$$
\begin{array}{ll}
y_{\mathrm{ij}} & \text { é o valor observado do tratamento } \mathrm{i}(\mathrm{i}=1,2, \ldots, \\
& \mathrm{v}=100), \text { na repetição } \mathrm{j}(\mathrm{j}=1,2, \ldots, \mathrm{r}=2, \text { ou } 3) ; \\
\mu & \text { é uma constante inerente a todas as observações; } \\
\mathrm{t}_{\mathrm{i}} & \text { é o efeito do tratamento } \mathrm{i} ; \\
\mathrm{r}_{\mathrm{j}} & \text { é o efeito da repetição } \mathrm{j} ; \mathrm{e} \\
\mathrm{e}_{\mathrm{ij}} & \text { é o erro aleatório associado à observação } \mathrm{y}_{\mathrm{ij}}
\end{array}
$$

\begin{tabular}{|c|c|c|c|c|}
\hline FV & GL & SQ & QM & $\mathrm{E}(\mathrm{QM})$ \\
\hline Repetições & $\mathrm{r}-1$ & SQRep.=R $(\alpha / \mu)$ & $\mathrm{Q}_{4}$ & $\sigma^{2}+k \sigma_{b}^{2}+v \sigma_{r}^{2}$ \\
\hline Blocos/Rep. (não-ajustados) & $\mathrm{r}(\mathrm{k}-1)$ & SQB $/$ Rep.(não ajustados) $=R(\beta / \mu, \alpha)$ & $\mathrm{Q}_{3}$ & $\sigma^{2}+\sigma_{g}^{2}+k \sigma_{b}^{2}$ \\
\hline Tratamentos (ajustados) & $\mathrm{v}-1$ & SQTrat. (ajustados) $=\mathrm{R}(\tau / \mu, \alpha, \beta)$ & $\mathrm{Q}_{2}$ & $\sigma^{2}+\left(\frac{k}{k+1}\right) r \sigma_{g}^{2}$ \\
\hline Resíduo & $(\mathrm{k}-1)(\mathrm{rk}-\mathrm{k}-1)$ & SQRes. $=Y^{\prime} Y-R(\mu, \tau, \alpha, \beta)$ & $\mathrm{Q}_{1}$ & $\sigma^{2}$ \\
\hline Total & rv-1 & SQTot. $=Y^{\prime} Y-R(\mu)$ & & \\
\hline
\end{tabular}

Para realizar esse tipo de análise, considerou-se cada repetição do látice como sendo um bloco completo (inclui todos os tratamentos) e utilizou-se o modelo usual para análise de experimentos em blocos casualizados completos.

Quadro 1. Esquema da análise da variância e esperanças dos quadrados médios da análise intrablocos do látice, com tratamentos ajustados e blocos dentro de repetições não-ajustados (não-ajust.), considerando o modelo aleatório 
Para a obtenção das esperanças matemáticas dos quadrados médios, apresentadas no quadro 2, adotaram-se as seguintes pressuposições:

a) $t_{i} \sim \operatorname{NID}\left(0, \sigma^{2}\right)$;

b) $\mathrm{r}_{\mathrm{j}} \sim \mathrm{NID}\left(0, \sigma_{\mathrm{r}}^{2}\right)$;

c) $\mathrm{e}_{\mathrm{ij}} \sim \mathrm{NID}\left(0, \sigma^{2}\right)$; e

d) $t_{i}, r_{j}$ e $e_{i j}$ independentes.

A partir das esperanças matemáticas dos quadrados médios apresentadas no quadro 2, obtiveram-se os estimadores dos componentes da variância, dados por:

$$
\hat{\sigma}^{2}=Q_{1}
$$

$\mathrm{e}$

$$
\hat{\sigma}_{\mathrm{g}}^{2}=\frac{\mathrm{Q}_{2}-\mathrm{Q}_{1}}{\mathrm{r}}
$$

\subsection{Análise intrablocos com tratamentos não-ajustados e blocos dentro de repetições ajustados - terceira análise}

Para realizar a análise individual intrablocos do látice com tratamentos não-ajustados e blocos dentro de repetições ajustados, utilizou-se o mesmo modelo estatístico e pressuposições adotados para realização da análise intrablocos com tratamentos ajustados e blocos dentro de repetições não-ajustados (primeira análise), diferindo desta apenas na decomposição ortogonal da soma de quadrados de parâmetros que, para o presente caso foi assim expressa:

$$
\begin{array}{r}
\mathrm{R}(\mu, \tau, \alpha, \beta)=\mathrm{R}(\mu)+\mathrm{R}(\alpha / \mu)+\mathrm{R}(\tau / \mu, \alpha)+ \\
\mathrm{R}(\beta / \mu, \tau, \alpha),
\end{array}
$$

Quadro 2. Esquema da análise da variância e esperanças dos quadrados médios, para análise do látice como blocos casualizados completos, considerando o modelo aleatório

\begin{tabular}{lcccc}
\hline FV & GL & SQ & QM & $\mathrm{E}(\mathrm{QM})$ \\
Repetições & $\mathrm{r}-1$ & SQRep. & $\mathrm{Q}_{3}$ & $\sigma^{2}+\mathrm{v} \sigma_{\mathrm{r}}^{2}$ \\
Tratamentos & $\mathrm{v}-1$ & SQTrat. & $\mathrm{Q}_{2}$ & $\sigma^{2}+\mathrm{r} \sigma_{\mathrm{g}}^{2}$ \\
Resíduo & $(\mathrm{r}-1)(\mathrm{v}-1)$ & SQRes. & $\mathrm{Q}_{1}$ & $\sigma^{2}$ \\
\hline
\end{tabular}

a partir da qual obteve-se o esquema da análise da variância com as respectivas esperanças matemáticas dos quadrados médios apresentado no quadro 3.

A partir do quadro 3, obtiveram-se os estimadores dos componentes da variância, dados por:

$$
\begin{aligned}
& \hat{\sigma}^{2}=Q_{1} \\
& \hat{\sigma}_{\mathrm{g}}^{2}=\left[\frac{\mathrm{Q}_{2}-\mathrm{Q}_{1}}{\mathrm{r}}\right]-\left[\frac{\mathrm{Q}_{3}-\mathrm{Q}_{1}}{(\mathrm{k}+1)(\mathrm{r}-1)}\right] \\
& \hat{\sigma}_{\mathrm{b}}^{2}=\frac{\mathrm{r}\left(\mathrm{Q}_{3}-\mathrm{Q}_{1}\right)}{\mathrm{k}(\mathrm{r}-1)}
\end{aligned}
$$

\subsection{Análise do látice como blocos casualizados} completos, utilizando as médias ajustadas da análise com recuperação da informação interblocos e tendo como quadrado médio do resíduo a variância efetiva média dessa mesma análise, com recuperação da informação interblocos - quarta análise

O primeiro procedimento para se realizar esta análise foi obter as médias ajustadas dos tratamentos da análise com recuperação da informação interblocos, procedimento este realizado através do método apresentado por PiMENTEL-Gomes (1990). Em seguida realizou-se a análise do látice como blocos casualizados completos, utilizando-se as médias ajustadas dos tratamentos da análise, com recuperação da informação interblocos e tendo como quadrado médio do resíduo a variância efetiva média dessa mesma análise do látice, com recuperação da informação interblocos.

As esperanças matemáticas dos quadrados médios, para esta análise, considerando o modelo aleatório, apresentadas no quadro 4 , foram simplesmente acopladas ao esquema de análise, como se fosse o modelo usual de análise de experimento em blocos casualizados completos, adotando as mesmas pressuposições desse modelo. Tal procedimento é idêntico ao adotado por VIANNA e SILVA (1978), sendo uma alternativa de análise aproximada, muito utilizada pelos melhoristas de plantas.

Quadro 3. Esquema da análise da variância e esperanças dos quadrados médios da análise intrablocos do látice, com tratamentos não-ajustados (não-ajust.) e blocos dentro de repetições ajustados (ajust.), considerando o modelo aleatório

\begin{tabular}{lclcc}
\hline FV & GL & \multicolumn{1}{c}{ SQ } & QM & E(QM) \\
Repetições & $\mathrm{r}-1$ & SQRep. $=\mathrm{R}(\alpha / \mu)$ & $\mathrm{Q}_{4}$ & $\sigma^{2}+\mathrm{k} \sigma_{\mathrm{b}}^{2}+\mathrm{v} \sigma_{\mathrm{r}}^{2}$ \\
Bloc/Rep(ajust.) & $\mathrm{r}(\mathrm{k}-1)$ & $\mathrm{SQB} /$ Rep.(ajust. $)=\mathrm{R}(\beta / \mu, \tau, \alpha)$ & $\mathrm{Q}_{3}$ & $\sigma^{2}+\left(\frac{\mathrm{r}-1}{\mathrm{r}}\right) \mathrm{k} \sigma_{\mathrm{b}}^{2}$ \\
Tratamentos(não-ajust.) & $\mathrm{v}-1$ & SQTrat.(não-ajust. $)=\mathrm{R}(\tau / \mu, \alpha)$ & $\mathrm{Q}_{2}$ & $\sigma^{2}+\left(\frac{\mathrm{k}}{\mathrm{k}+1}\right) \sigma_{\mathrm{b}}^{2}+\mathrm{r} \sigma_{\mathrm{g}}^{2}$ \\
Resíduo & $(\mathrm{k}-1)(\mathrm{rk}-\mathrm{k}-1)$ & SQRes. $=\mathrm{Y}^{\prime} \mathrm{Y}-\mathrm{R}(\mu, \tau, \alpha, \beta)$ & $\mathrm{Q}_{1}$ & $\sigma^{2}$ \\
\hline Total & $\mathrm{rv}-1$ & SQTot. $=\mathrm{Y}^{\prime} \mathrm{Y}-\mathrm{R}(\mu)$ & & \\
\hline
\end{tabular}


Quadro 4. Esquema da análise da variância e esperanças dos quadrados médios, para análise do látice como blocos casualizados completos, utilizando-se as médias ajustadas dos tratamentos (ajust.) da análise com recuperação da informação interblocos e tendo como quadrado médio do resíduo a variância efetiva média da mesma análise do látice, com recuperação da informação interblocos (quarta análise)

\begin{tabular}{lcccc}
\hline FV & GL & SQ & QM & $\mathrm{E}(\mathrm{QM})$ \\
\hline $\begin{array}{l}\text { Repetições } \\
\begin{array}{l}\text { Tratamentos } \\
\text { (ajust.) }\end{array}\end{array} \mathrm{r}-1^{\mathrm{v}-1}$ & $\begin{array}{l}\text { SQTrat. } \\
\text { (ajust.) }\end{array}$ & $\mathrm{Q}_{2}$ & $\sigma^{2}+\mathrm{r}_{\mathrm{g}}^{2}$ \\
Resíduo & $(\mathrm{k}-1)(\mathrm{rk}-\mathrm{k}-1)$ & - & $\mathrm{Q}_{1}^{*}$ & $\sigma^{2}$ \\
\hline
\end{tabular}

$$
\text { SQTrat.(ajust.)* }=r\left[\sum_{\mathrm{i}} \hat{\mathrm{m}}_{\mathrm{i}}-\frac{\left(\sum_{\mathrm{i}} \hat{\mathrm{m}}_{\mathrm{i}}\right)^{2}}{\mathrm{v}}\right]
$$

com $\hat{\mathrm{m}}_{\mathrm{i}}=$ "média ajustada" do tratamento i.

$\mathrm{Q}_{1}{ }^{*}$ é a variância efetiva média da análise do látice com recuperação da informação interblocos $\left(\mathrm{V}_{\mathrm{r}}^{\prime}\right)$, é dada por:

$$
\mathrm{Q}_{1} *=\mathrm{V}_{\mathrm{r}}^{\prime}=\left[1+\left(\frac{\mathrm{r}}{(\mathrm{r}-1)(\mathrm{k}+1)} \frac{\left(\mathrm{V}_{\mathrm{b}}-\mathrm{V}_{\mathrm{r}}\right)}{\mathrm{V}_{\mathrm{b}}}\right)\right] \mathrm{V}_{\mathrm{r}}
$$

em que

r é o número de repetições;

k é o número de parcelas em cada bloco;

$\mathrm{V}_{\mathrm{b}}$ é o quadrado médio da análise intrablocos para o efeito de blocos dentro de repetições (ajustado); e

$\mathrm{V}_{\mathrm{r}}$ é o quadrado médio do resíduo intrablocos.

A partir das esperanças matemáticas dos quadrados médios apresentadas no quadro 4 , obtiveram-se os estimadores dos componentes de variância, dados por:

$$
\hat{\sigma}^{2}=Q_{1} *
$$

e

$$
\hat{\sigma}_{\mathrm{g}}^{2}=\frac{\mathrm{Q}_{2}-\mathrm{Q}_{1} *}{\mathrm{r}}
$$

\subsection{Eficiência relativa}

A eficiência relativa dos experimentos montados em látice da análise com recuperação da informação interblocos, em relação aos blocos casualizados completos, foi calculada pela fórmula:

$$
\mathrm{Ef}=\frac{\mathrm{QMR}}{\mathrm{V}_{\mathrm{r}}^{\prime}} \times 100
$$

em que

$\mathrm{V}^{\prime}{ }_{\mathrm{r}}=$ variância efetiva média da análise do látice com recuperação de informação interblocos;
QMR = quadrado médio do resíduo da análise do látice como blocos casualizados completos.

\section{RESULTADOS E DISCUSSÃO}

Nos quadros 5 a 8 encontram-se apresentadas as estimativas médias dos componentes da variância devido a efeitos de tratamentos $\left(\hat{\sigma}_{\mathrm{g}}^{2}\right)$, residual $\left(\hat{\sigma}^{2}\right)$ e de blocos dentro de repetições $\left(\hat{\sigma}_{b}^{2}\right)$, com seus respectivos erros padrões, obtidas pelas quatro alternativas de análise, nas duas situações estudadas, alta e baixa eficiência relativa do látice em relação aos blocos casualizados completos, para ambos os delineamentos, látice simples e triplo.

Pode-se observar que os estimadores do componente da variância devido a efeitos de tratamentos $\hat{\sigma}_{g^{\prime}}^{2}$ obtidos a partir da primeira, segunda e terceira análises, mostram-se não-tendenciosos, independentemente da eficiência relativa do látice em relação aos blocos casualizados completos ser alta ou baixa, o mesmo não ocorrendo com a quarta análise, que se revelou tendenciosa em ambos os casos. Quando a

Quadro 5. Estimativas médias dos componentes de variância devido a efeitos de tratamentos, residuais e de blocos dentro de repetições, com seus respectivos erros padrões, obtidas para os experimentos com duas repetições e alta eficiência relativa $(\overline{\mathrm{E} f}=179,34 \%)$

\begin{tabular}{lcrc}
\hline Análise & $\hat{\sigma}_{\mathrm{g}}^{2}$ & $\hat{\sigma}^{2}$ & $\hat{\sigma}_{\mathrm{b}}^{2}$ \\
\hline 1 $^{\mathrm{a}}$ Análise & $196,45 \pm 36,36$ & $95,34 \pm 15,17$ & $109,06 \pm 45,85$ \\
2 $^{\mathrm{a}}$ Análise & $196,96 \pm 42,37$ & $193,97 \pm 40,79^{*}$ & - \\
3 $^{\mathrm{a}}$ Análise & $196,96 \pm 42,37$ & $95,34 \pm 15,17$ & $108,50 \pm 43,00$ \\
4 $^{\mathrm{a}}$ Análise & $238,90 \pm 43,63^{*}$ & $109,66 \pm 17,07^{*}$ & - \\
Parâmetro & 195 & 95 & 110 \\
\hline
\end{tabular}

* significativamente diferente do parâmetro $(\mathrm{P}<0,01)$.

Quadro 6. Estimativas médias dos componentes da variância devido a efeitos de tratamentos, residuais e de blocos dentro de repetições, com seus respectivos erros padrões, para os experimentos com duas repetições e baixa eficiência relativa $(\overline{E f}=98,65 \%)$

\begin{tabular}{lccc} 
Análise & $\hat{\sigma}_{\mathrm{g}}^{2}$ & $\hat{\sigma}^{2}$ & $\hat{\sigma}_{\mathrm{b}}^{2}$ \\
\hline 1 $^{\mathrm{a}}$ Análise & $195,02 \pm 36,38$ & $95,34 \pm 14,54$ & $0,21 \pm 10,11$ \\
2 $^{\mathrm{a}}$ Análise & $194,91 \pm 35,73$ & $95,34 \pm 13,59$ & - \\
$3^{\mathrm{a}}$ Análise & $194,91 \pm 35,73$ & $95,34 \pm 14,54$ & $0,34 \pm 3,32$ \\
$4^{\mathrm{a}}$ Análise & $164,18 \pm 35,79^{*}$ & $97,11 \pm 14,23^{*}$ & - \\
Parâmetro & 195 & 95 & 0 \\
\hline
\end{tabular}

* significativamente diferente do parâmetro $(\mathrm{P}<0,01)$. 
Quadro 7. Estimativas médias dos componentes da variância devido a efeitos de tratamentos, residuais e de blocos dentro de repetições, com seus respectivos erros padrões, obtidas para os experimentos com três repetições e alta eficiência relativa $(\overline{\mathrm{E}} \mathrm{f}=185,36 \%)$

\begin{tabular}{lccc}
\hline Análise & $\hat{\sigma}_{\mathrm{g}}^{2}$ & $\hat{\sigma}^{2}$ & $\hat{\sigma}_{\mathrm{b}}^{2}$ \\
\hline 1 $^{\mathrm{a}}$ Análise & $193,43 \pm 33,88$ & $95,45 \pm 9,99$ & $112,91 \pm 38,58$ \\
2 $^{\mathrm{a}}$ Análise & $194,79 \pm 38,65$ & $196,74 \pm 32,54^{*}$ & - \\
3 $^{\mathrm{a}}$ Análise & $194,79 \pm 38,65$ & $95,45 \pm 9,99$ & $111,48 \pm 34,99$ \\
4$^{\mathrm{a}}$ Análise & $224,79 \pm 39,45^{*}$ & $106,86 \pm 11,02^{*}$ & - \\
Parâmetro & 195 & 95 & 110 \\
\hline
\end{tabular}

- significativamente diferente do parâmetro $(\mathrm{P}<0,01)$.

Quadro 8. Estimativas médias dos componentes da variância devido a efeitos de tratamentos, residuais e de blocos dentro de repetições, com seus respectivos erros padrões, obtidas para os experimentos com três repetições e baixa eficiência relativa $(\overline{\mathrm{E} f}=98,99 \%)$

\begin{tabular}{lccc}
\hline Análise & $\hat{\sigma}_{\mathrm{g}}^{2}$ & $\hat{\sigma}^{2}$ & $\hat{\sigma}_{\mathrm{b}}^{2}$ \\
\hline $1^{\mathrm{a}}$ Análise & $196,51 \pm 33,21$ & $95,13 \pm 10,44$ & $-0,23 \pm 9,20$ \\
$2^{\mathrm{a}}$ Análise & $196,27 \pm 32,53$ & $95,15 \pm 9,70$ & - \\
3 $^{\mathrm{a}}$ Análise & $196,27 \pm 32,53$ & $95,13 \pm 10,44$ & $0,02 \pm 4,16$ \\
$4^{\mathrm{a}}$ Análise & $194,89 \pm 32,60$ & $98,51 \pm 11,92^{*}$ & - \\
Parâmetro & 195 & 95 & 0 \\
\hline
\end{tabular}

* significativamente diferente do parâmetro $(\mathrm{P}<0,01)$.

eficiência relativa do látice em relação aos blocos casualizados completos foi alta, esta análise superestimou o referido componente de variância, e, quando a eficiência foi baixa, a análise o subestimou, confirmando as observações de SILVa (1997) a respeito de látices simples 6 × 6 . Observa-se que, quando se utilizaram três repetições do delineamento em látice, as estimativas desse componente, obtidas pela quarta análise, na situação de baixa eficiência relativa, foram estatisticamente iguais ao componente estimado, indicando que para um maior número de repetições e baixa eficiência, este estimador seja não-tendencioso. No entanto, estudos mais aprofundados serão necessários para confirmar tal fato.

Apesar de a primeira, segunda e terceira análises terem-se mostrado não-tendenciosas, quanto às estimativas do componente $\hat{\sigma}_{\mathrm{g}^{\prime}}^{2}$ observa-se que a primeira análise mostrou-se mais eficiente que as demais, a saber: $135 \%$ em relação à segunda e terceira análises e 143,5\% em relação à quarta análise (para os experimentos com duas repetições), $130 \%$ em relação à segunda e terceira análises e 135\% em relação à quarta análise (para os experimentos com três repetições) quando a eficiência relativa foi alta. Quando a eficiência relativa foi baixa (menor que $100 \%$ ), a segunda e terceira análises mostram-se ligeiramente mais eficientes: 106\% e 100,3\% em relação à primeira e quarta análises, respectivamente (para os experimentos com duas repetições), e 104\% e 100,4\% em relação à primeira e quarta análises, respectivamente (para os experimentos com três repetições).

Observa-se que, tanto a acurácia quanto a precisão dos estimadores do componente de variância residual $\left(\hat{\sigma}^{2}\right)$ estão intimamente relacionadas à eficiência relativa do delineamento em látice, em relação aos blocos casualizados completos, pois, quando essa eficiência foi alta, os estimadores obtidos pela primeira e terceira análises (análises intrablocos do látice) mostraram-se não-tendenciosos e mais eficientes que os demais, sendo sua eficiência, em relação à segunda análise, de $722 \%$ e, em relação à quarta, de $126 \%$, nos experimentos com duas repetições, e de $1.060 \%$ e $121 \%$ em relação à primeira e quarta análises, respectivamente, para os experimentos com três repetições. Quando a eficiência relativa do látice foi baixa, somente o estimador obtido pela quarta análise foi tendencioso, sendo o estimador mais eficiente, neste caso, o obtido pela segunda análise, que apresentou uma eficiência relativa da ordem de $114 \%$, em relação à primeira e terceira análises e de 109\%, em relação à quarta, nos experimentos com duas repetições e de $152 \%$ em relação à primeira e terceira análises e 151\% em relação à quarta (nos experimentos com três repetições).

Observa-se, ainda, que o viés do estimador obtido pela segunda análise, em relação ao obtido pela primeira ou terceira, é igual a $\mathrm{k} /(\mathrm{k}+1) \hat{\sigma}_{\mathrm{b}}^{2} 3^{\mathrm{a}}$ análise, confirmando a relação apresentada por SILVA (1997), $\hat{\sigma}_{2^{a} \text { análise }}^{2}=\hat{\sigma}_{1^{a} \text { ou } 3^{a} \text { análise }}^{2}$ o que indica que, quanto maior for o efeito de blocos dentro de repetições e, conseqüentemente, maior a eficiência relativa do delineamento em látice em relação aos blocos casualizados completos, mais tendenciosas serão as estimativas obtidas por esta análise.

\section{CONCLUSÕES}

1. Os estimadores do componente da variância devido a efeitos de tratamentos, obtidos pela primeira, segunda e terceira análises foram não-tendenciosos. Na situação de alta eficiência relativa do látice, o estimador obtido pela primeira análise foi o mais eficiente, e na situação de baixa eficiência relativa, o estimador mais eficiente foi o obtido pela segunda análise. 
2. O estimador do componente da variância devido a efeito de tratamentos, obtido pela quarta análise, mostrou-se tendencioso, superestimando o referido componente nas situações de alta eficiência relativa, e, em geral, subestimando-o nas situações de baixa eficiência relativa.

3. O estimador do componente da variância residual, obtido pela primeira e terceira análises (análises intrablocos do látice), foi não-tendencioso em ambas as situações (alta e baixa eficiência), o que não ocorreu com o obtido pela segunda análise (análise como blocos casualizados completos) que foi não-tendencioso apenas nas situações de baixa eficiência.

4. Na situação de alta eficiência relativa o estimador do componente da variância residual mais eficiente foi o obtido pela primeira e terceira análises, enquanto no caso de baixa eficiência, o mais eficiente foi o obtido pela segunda análise.

5. Os resultados acima sugerem que se deve utilizar o modelo de análise intrablocos de experimentos em látice, para estimar componentes da variância sempre que a eficiência relativa do delineamento em látice, em relação ao delineamento em Blocos Completos Casualizados, for superior a $100 \%$ e, em caso contrário, deve-se optar pelo modelo de análise em blocos casualizados completos, não devendo ser recomendada a quarta alternativa de análise em nenhuma das duas situações.

\section{AGRADECIMENTOS}

Os autores agradecem à Fundação de Amparo à Pesquisa do Estado de Minas Gerais (FAPEMIG), pelo financiamento deste projeto.

\section{REFERÊNCIAS BIBLIOGRÁFICAS}

DACHS, J.N.W. Estatística Computacional. Rio de Janeiro: LTC Editora,1988, 236p.

PIMENTEL-GOMES, F. Curso de estatística experimental 13.ed. Piracicaba: Livraria Nobel, 1990. 468p.

SILVA, H.D. Análise de experimentos em látice quadrado ("Square Lattice") com ênfase em componentes de variância e aplicações no melhoramento genético vegetal. Viçosa, 1997. 139p. Dissertação (Mestrado em Genética e Melhoramento) - Universidade Federal de Viçosa.

VIANA, J.M.S. Análise individual e conjunta intrablocos de experimentos em Látice Quadrado ("Square Lattice"), com aplicação no melhoramento genético. Viçosa, 1993. 89p. Monografia (Monografia de Genética e Melhoramento) - Universidade Federal de Viçosa.

VIANNA, R.T.; SILVA, J.C. Comparação de três métodos estatísticos de análise de experimentos em "Látice" em milho (Zea mays L.). Experimentiae, Viçosa, v.24, n.2, p. 21-41, 1978.

YATES, F.A. A new method of arranging variety trials involving a large number of varieties. Journal of Agricultural Science, v.26, p. 424-455, 1936. 